\title{
CORRESPONDENCE \\ How much do parents actually use their smartphones? Pilot study comparing self-report to passive sensing
}

\author{
Pediatric Research (2019) 86:416-418; \\ https://doi.org/10.1038/s41390-019-0452-2
}

\section{INTRODUCTION}

Parent smartphone use has been associated with decreased responsiveness to children, ${ }^{1}$ fewer verbal and nonverbal interactions, $^{2}$ and higher ratings of child externalizing behavior. ${ }^{3}$ However, existing studies are limited by use of brief (10-15 min) observations of parents using smartphones ${ }^{1,4}$ or rely on parent self-report, ${ }^{3}$ which may be biased by social desirability. In addition, self-report of smartphone use may be inaccurate due to the brief, intermittent ways users interact with mobile devices, ${ }^{5}$ which makes recall difficult. ${ }^{6}$

The purpose of this pilot study was to improve upon these measurement limitations by implementing a novel approachpassive mobile sensing -in order to objectively assess parent smartphone use over several days. Passive mobile sensing harnesses data already collected by mobile devices, such as application ("app") or battery usage, and generates usage logs that are transmitted to cloud-based servers or can be emailed to research teams. This approach has promise for studying daily patterns of participant behavior, ${ }^{7}$ and has been used to document associations between higher iPhone usage and depression symptoms in adults; ${ }^{8}$ however, this method has not been used in research studies of parenting or child well-being. It is also a potential powerful research tool for studies examining mobile media use and health outcomes such as obesity and sleep, which have been linked to heavy media use in prior research. ${ }^{9}$

The aims of this pilot study were to: (1) test the feasibility and acceptability of passive sensing as a measure of smartphone use; (2) describe smartphone usage by parents of young children over the course of several days, including duration, checking frequency, and app content (e.g., social media, email); and (3) examine whether parents are accurate self-reporters of daily smartphone duration and checking frequency, compared to objective passive sensing output.

\section{METHOD}

Parents took part in a 2-week study that involved completing surveys at enrollment, installing a passive sensing app, and completing a media use recall survey upon app uninstallation. Parents were compensated US\$50 for their participation. The study was approved by the University of Michigan Medical School Institutional Review Board.

We recruited English-speaking parents via a university research participant registry to participate in a study in which they would "download a mobile device use tracking application on their smartphone." Parents were eligible to participate if they: (1) had a child age 12 months to 5 years, (2) were biological or custodial parent, (3) lived with the child at least 5 days per week, (4) and used an Android smartphone or iPhone (the two main smartphone operating systems). ${ }^{10}$ After providing informed consent, 87 parents completed surveys reporting their demographic characteristics and depression symptoms ${ }^{11}$ and installed a passive sensing app on their smartphone (Moment for iPhones, Minuku ${ }^{12}$ for Android) for 14 days (see Supplementary Appendix for additional information about passive sensing apps).

Moment is a commercially available app used in prior research, which records screen on/off status to provide daily estimates of smartphone use duration (i.e., minutes screen is on) and number of pickups (i.e., screen turns from off to on). Moment also prompts users to take a screenshot of their battery usage, which undergoes image recognition to generate output of the average minutes/day each app was used. Users can export their usage data in json file format, which we instructed them to email to our secure laboratory email account.

Minuku is a prototype passive sensing app developed at the University of Michigan (PI: Chang, Newman) designed to query the Android UsageStatsManager library (https://developer.android. com/reference/android/app/usage/UsageStatsManager) every $5 \mathrm{~s}$ to obtain app usage history and statistics. Data are automatically sent to cloud-based storage, from which they are downloaded by the research team. We opted to use the Minuku app because of its simple data structure and because commercially available app trackers for Android devices do not allow exporting data to research teams. Minuku datafiles, which include screen on/off status, name of app last running in the foreground, and timestamp of last app usage, were automatically transmitted to a secure cloud-based server and downloaded by the researchers after 14 days. We calculated the difference between each timestamp and summed all instances when the screen was on to obtain overall duration of usage, and duration of each specific app, per day. Each time the screen status changed from off to on was considered a pickup, from which we calculated daily pickup counts. We then calculated average daily duration of smartphone use, average daily pickup frequency, and average daily minutes of eight different app categories for each participant.

We trained two research assistants to reliably categorize each app name into categories based on prior human-computer interaction research: ${ }^{13}$ social media (e.g., Facebook, Twitter, Pinterest); entertainment (e.g., on-demand streaming video services, podcasts), task-oriented apps (e.g., clock, calendar, weather); communication apps (e.g., texting, calls); Internet browser; email; photos/photoediting; and shopping (kappa = 0.77).

After 14 days of running either app on their smartphone, parents were instructed to uninstall the app and complete an online survey of family media use, including the questions: "Thinking about yourself yesterday, how much time did you personally spend using a smartphone?" and "How many times do you check your smartphone on a typical day?" We categorized parents as accurate for duration if their self-report was within $60 \mathrm{~min}$ of the average daily duration calculated from app output, 
and as accurate for checking frequency if their app output pickup frequency fell within the range they self-reported. Because smartphone usage data was positively skewed, we used Spearman's correlations, Wilcoxon's rank-sum tests, and Kruskal-Wallis tests to examine associations between smartphone usage variables, parent/household characteristics, and accuracy of duration and checking frequency self-report.

\section{RESULTS}

Acceptability of passive sensing was high among participants who expressed interest in the study. No participants refused to install a passive sensing app, and none expressed concern about data privacy protections, which were described thoroughly during the informed consent process.

Feasibility of passive sensing with Moment was good, as parents reported no difficulties downloading it from the app store. However, this app requires participants to export their datafile to research teams via email, which three participants failed to do (10\%). Feasibility of data collection with Minuku was lower, as 26 participants (45\%) had incomplete data appear on the server, which we monitored on a daily basis. This high rate of missing data with Android phones was presumed due to built-in battery optimization applications, specific to certain manufacturers, which disable background processes such as passive sensing apps.

The final analytic sample comprised 58 parents ( 26 iPhone and 32 Android); included participants did not differ from excluded participants in terms of demographic characteristics. Participant characteristics and average daily smartphone usage are shown in Table 1. Average daily smartphone duration was 234.4 (SD 157.8) minutes, average checking frequency was 66.8 (SD 40.7) times per day, and the highest duration app categories were task-related apps, social media, communication, and entertainment. High duration of smartphone use was most highly correlated with more entertainment use $(R=0.62, p<0.0001)$, task-oriented use $(R=$ $0.80, p<0.0001)$, communication $(R=0.40, p=0.005)$, and social media use $(R=0.69, p<0.0001)$, but was not correlated with average checking frequency $(R=-0.01, p=0.90)$.

Overall, few parents accurately self-reported their daily smartphone duration and checking, with most underreporting and some overreporting (Table 2). Parents from lower income households $(p=0.037)$, with higher depression symptoms $(p=0.002)$, higher daily duration of smartphone use $(p=0.0003)$, or more use of task-oriented ( $p=0.0012)$ or browser apps $(p=0.001)$ were less accurate in their self-reported duration. Checking frequency selfreport accuracy was not associated with parent characteristics. Although Moment provides visual feedback about smartphone usage, iPhone users were no more accurate in self-report of duration or checking than Android users.

\section{DISCUSSION}

This is the first study to use mobile passive sensing to measure parent smartphone usage and compare directly measured mobile device usage to self-report. This preliminary work demonstrates the feasibility and utility of this novel methodology, and suggests that parent-reported recall of their own smartphone use may not be accurate.

Although our results are exploratory and need to be replicated in larger samples, several important research implications are highlighted by this work. First, mobile data collection was highly acceptable to parents, as it conferred low participant burden and our informed consent process transparently outlined how their data would be collected, analyzed, and stored. Feasibility varied by type of smartphone: data collection from iPhones required the participant to take screen shots and export the Moment datafile to the research team, which conferred a slightly higher participant burden and led to missing data in three participants. Although
Table 1. Participant characteristics and daily smartphone usage derived from Minuku and Moment output

\begin{tabular}{|c|c|c|}
\hline Participant characteristics $(n=58)$ & $\begin{array}{l}\text { Mean (SD) or } \\
N(\%)\end{array}$ & Range \\
\hline Parent age & $33.4(5.2)$ & $26.0-60.4$ \\
\hline Child age (years) & $3.12(1.30)$ & $1.05-5.93$ \\
\hline \multicolumn{3}{|l|}{ Parent sex } \\
\hline Male & $7(12.1)$ & \\
\hline Female & $51(87.9)$ & \\
\hline \multicolumn{3}{|l|}{ Child sex } \\
\hline Male & $31(53.5)$ & \\
\hline Female & $27(46.6)$ & \\
\hline \multicolumn{3}{|l|}{ Parent race/ethnicity } \\
\hline White/non-Hispanic & 45 (77.6) & \\
\hline Other & $13(22.4)$ & \\
\hline \multicolumn{3}{|l|}{ Parent education } \\
\hline Less than college & $15(27.8)$ & \\
\hline College graduate & $39(72.2)$ & \\
\hline \multicolumn{3}{|l|}{ Parent employment status } \\
\hline Unemployed/stay at home parent & $21(36.2)$ & \\
\hline Part-time/full-time job & $37(63.8)$ & \\
\hline \multicolumn{3}{|l|}{ Parent marital status } \\
\hline Married/partner & $53(91.4)$ & \\
\hline Single/separated/divorced & $5(8.6)$ & \\
\hline Income-to-needs ratio & $3.50(2.00)$ & $0.20-7.80$ \\
\hline CES-D score $\geq 16$ & $13(22.4)$ & \\
\hline \multicolumn{3}{|l|}{ Type of smartphone } \\
\hline iPhone & $26(44.8)$ & \\
\hline Android & $32(55.2)$ & \\
\hline Smartphone use variables & Mean (SD) & Range \\
\hline Total duration (min/day) & $234.4(157.8)$ & $38.4-861.4$ \\
\hline Daily checking frequency & $66.8(40.7)$ & $23.6-245.8$ \\
\hline \multicolumn{3}{|l|}{ App usage (min/day) } \\
\hline Social media & $49.3(50.0)$ & $0-215.1$ \\
\hline $\begin{array}{l}\text { Entertainment apps (e.g., YouTube, } \\
\text { Netflix) }\end{array}$ & $41.4(66.2)$ & $0-316.1$ \\
\hline $\begin{array}{l}\text { Task-related apps (e.g., maps, } \\
\text { security, weather) }\end{array}$ & $55.0(71.6)$ & $4.3-437.4$ \\
\hline $\begin{array}{l}\text { Communication apps (e.g., texting, } \\
\text { phone calls) }\end{array}$ & $46.1(33.7)$ & $4.4-172.0$ \\
\hline Browser & $31.2(33.6)$ & $0-157.7$ \\
\hline Email & $7.54(11.3)$ & $0-73.5$ \\
\hline Photo/gallery/video-editing & $4.11(5.13)$ & $0-23.7$ \\
\hline Shopping & $6.32(10.8)$ & $0-50.6$ \\
\hline
\end{tabular}

Minuku data collection was automatic, we had more missing data due to updates in Android smartphones' design and operating systems, including battery optimizing apps that scan for and disable background processes. However, participant characteristics were similar between those whose data we included vs. excluded. Given the detailed, timestamped data generated by Minuku and other passive sensing apps for Android, this approach holds promise for highly accurate assessment of parent and child mobile media use with low participant burden, and we therefore continue to develop solutions for Android-based data collection with lower rates of missing data. In addition, there have been recent requests for technology companies to share participant 
Table 2. Comparison of self-reported daily duration of smartphone usage and checking frequency, compared to actual mobile app output

\begin{tabular}{|c|c|c|}
\hline $\begin{array}{l}\text { Accuracy of smartphone daily } \\
\text { duration recall }\end{array}$ & $N(\%)$ & $\begin{array}{l}\text { Actual average daily } \\
\text { duration, mean (SD) } \\
\text { (min/day) }\end{array}$ \\
\hline Accurate & $15(39.5)$ & $153.8(80.0)$ \\
\hline Underreport & $18(47.4)$ & $352.3(191.4)$ \\
\hline Overreport & $5(13.2)$ & 210.5 \\
\hline $\begin{array}{l}\text { Accuracy of smartphone daily } \\
\text { checking frequency recall }^{\mathrm{b}}\end{array}$ & $N(\%)$ & $\begin{array}{l}\text { Actual phone checks } \\
\text { per day, mean (SD) }\end{array}$ \\
\hline Accurate & $16(32.0)$ & $64.6(32.0)$ \\
\hline Underreport & $27(54.0)$ & $77.6(47.4)$ \\
\hline Overreport & $7(14.0)$ & $33.4(7.6)$ \\
\hline $\begin{array}{l}\text { Self-reported daily checking } \\
\text { frequency category }\end{array}$ & $N(\%)$ & $\begin{array}{l}\text { Actual phone checks } \\
\text { per day, mean (SD) }\end{array}$ \\
\hline Less than 10 times & $3(5.9)$ & $52.3(22.6)$ \\
\hline 10-20 times & $8(15.7)$ & $41.5(16.1)$ \\
\hline 20-40 times & $13(25.5)$ & $65.9(42.7)$ \\
\hline $40-60$ times & $15(29.4)$ & $62.4(31.5)$ \\
\hline $60-100$ times & $10(19.6)$ & $77.6(17.0)$ \\
\hline $100-150$ times & $2(3.9)$ & $191.8(76.4)$ \\
\hline \multicolumn{3}{|c|}{$\begin{array}{l}\text { aSelf-report categorized as accurate if within } 60 \text { min of actual average daily } \\
\text { duration; } n=38 \text { because } 20 \text { participants were missing data on self- } \\
\text { reported duration } \\
\text { bSelf-report categorized as accurate if within the self-report checking } \\
\text { frequency category; } n=50 \text { because } 1 \text { participant was missing data on } \\
\text { actual checking frequency and } 7 \text { participants were missing data on self- } \\
\text { reported checking frequency }\end{array}$} \\
\hline
\end{tabular}

usage data directly with researchers, ${ }^{14}$ which would be the simplest approach to facilitate more accurate measurement of mobile media and health behaviors.

Most importantly, we found that most parents underreport their smartphone duration and checking frequency, which varies with participant characteristics such as income and depression symptoms, and thus may introduce bias into studies relying on smartphone usage self-report. Underreporting may be due to the more intermittent or immersive experiences users have with mobile technology, whose duration may be more difficult to recall. Therefore, app-based mobile sensing may be an important tool for researchers hoping to measure duration, timing, and content of smartphone usage in clinical or behavioral research. Accurate measurement of everyday media experiences is especially important in light of recent calls for higher quality evidence regarding media effects on child health. ${ }^{15}$

\section{ACKNOWLEDGEMENTS}

Dr. Radesky is supported by K23HD092626 from NICHD.

\section{AUTHOR CONTRIBUTIONS}

N.Y. made substantial contributions to data acquisition and analysis, drafted the article, and approved the final version. H.M.W. made substantial contributions to design of the study, analysis and interpretation of data, revised the article critically for important intellectual content, and approved the final version. R.B. made substantial contributions to design of the study and data acquisition, revised the article critically for important intellectual content, and approved the final version. M.W.N. made substantial contributions to design of the study and data acquisition, revised the article critically for important intellectual content, and approved the final version. Y.-J. C. made substantial contributions to data acquisition, revised the article critically for important intellectual content, and approved the final version. J.S.R. designed the pilot study, oversaw data acquisition and analysis, interpreted the data, revised the article critically for important intellectual content, and approved the final version.

\section{ADDITIONAL INFORMATION}

The online version of this article (https://doi.org/10.1038/s41390-019-0452-2) contains supplementary material, which is available to authorized users.

Competing interests: The authors declare no competing interests.

Publisher's note: Springer Nature remains neutral with regard to jurisdictional claims in published maps and institutional affiliations.

Nalingna Yuan ${ }^{1}$, Heidi M. Weeks' ${ }^{2}$, Rosa Ball ${ }^{1}$, Mark W. Newman ${ }^{3}$, Yung-Ju Chang ${ }^{4}$ and Jenny S. Radesky ${ }^{1}$

${ }^{1}$ Department of Pediatrics, Division of Developmental Behavioral Pediatrics, University of Michigan Medical School, Ann Arbor, MI, USA; ${ }^{2}$ Department of Nutritional Sciences, University of Michigan School of Public Health, Ann Arbor, MI, USA; ${ }^{3}$ School of Information, University of Michigan, Ann Arbor, MI, USA and ${ }^{4}$ Department of Computer Science, National Chiao Tung University, Hsinchu, Taiwan Correspondence: Jenny S. Radesky (jradesky@umich.edu)

\section{REFERENCES}

1. Hiniker, A. et al. (eds) Texting while parenting: How adults use mobile phones while caring for children at the playground. In Proc. 33rd Annual ACM Conference on Human Factors in Computing Systems (ACM, Seoul, South Korea, 2015).

2. Radesky, J. et al. Maternal mobile device use during a structured parent-child interaction task. Acad. Pediatr. 15, 238-244 (2015).

3. McDaniel, B. T. \& Radesky, J. S. Technoference: parent distraction with technology and associations with child behavior problems. Child Dev. 89, 100-109 (2018).

4. Radesky, J. S. et al. Patterns of mobile device use by caregivers and children during meals in fast food restaurants. Pediatrics 133, e843-e849 (2014).

5. Oulasvirta, A., Tamminen, S., Roto, V. \& Kuorelahti J. (eds) Interaction in 4-second bursts: the fragmented nature of attentional resources in mobile $\mathrm{HCl}$. In Proc. SIGCHI Conference on Human Factors in Computing Systems (ACM, Portland, OR, 2005)

6. Burns, J. J. \& Anderson, D. R. Attentional inertia and recognition memory in adult television viewing. Commun. Res. 20, 777-799 (1993).

7. Harari, G. M. et al. Using smartphones to collect behavioral data in psychological science: opportunities, practical considerations, and challenges. Perspect. Psychol. Sci. 11, 838-854 (2016).

8. Elhai, J. D., Tiamiyu, M. F., Weeks, J. W., Levine, J. C., Picard, K. J., Hall, B. J. Depression and emotion regulation predict objective smartphone use measured over one week. Pers. Individ. Dif. 15, 21-8 (2018).

9. Chassiakos, Y. L. R., Radesky, J., Christakis, D., Moreno, M. A. \& Cross, C. Children and adolescents and digital media. Pediatrics 138, e20162593 (2016).

10. Anderson, M. Technology Device Ownership: 2015-2016. http://www. pewinternet.org/2015/10/29/technology-device-ownership-2015/. Accessed 1 Apr 2019.

11. Radloff, L. S. The CES-D scale a self-report depression scale for research in the general population. Appl. Psychol. Meas. 1, 385-401 (1977).

12. Chang, Y.-J. Using and Collecting Annotated Behavioral Trace Data for Designing and Developing Context-Aware Application. Dissertation, University of Michigan. (2016).

13. Hiniker, A., Patel, S. N., Kohno, T. \& Kientz, J. A. (eds) Why would you do that? predicting the uses and gratifications behind smartphone-usage behaviors. Proc. 2016 ACM International Joint Conference on Pervasive and Ubiquitous Computing (ACM, Heidelberg, Germany, 2016).

14. Christakis, D. The challenges of defining and studying "Digital Addiction" in children. JAMA (2019). https://doi.org/10.1001/jama.2019.4690. [Epub ahead of print].

15. Makin, S. Searching for digital technology's effects on well-being. Nature 563, S138 (2018) 